\title{
The exposure buildup factor formulation in a slab and rectangle geometry by the $\mathrm{LTS}_{N}$ method
}

\section{Bárbara D. Amaral Rodriguez*}

Mechanical Engineering Pos-Graduation Program

Federal University of Rio Grande do Sul - UFRGS

Rua Sarmento Leite, 425 - sala 314

Porto Alegre, Rio Grande do Sul, Brazil

Fax: +55 5133164001

E-mail: barbara.arodriguez@gmail.com

*Corresponding author

\section{Marco Túllio de Vilhena}

Pure and Applied Mathematics Department Federal University of Rio Grande do Sul - UFRGS Avenida Bento Gonçalves 9500

Porto Alegre, Rio Grande do Sul, Brazil

Fax: +55 5133164001

E-mail: vilhena@mat.ufrgs.br

\section{Volnei Borges}

Mechanical Engineering Pos-Graduation Program Federal University of Rio Grande do Sul - UFRGS

Rua Sarmento Leite, 425 - sala 314

Porto Alegre, Rio Grande do Sul, Brazil

Fax: +55 5133164001

E-mail: borges@ufrgs.br

\section{Carlos Malamut}

Centro de Desenvolvimento da Tecnologia Nuclear Comissão Nacional de Energia Nuclear

Belo Horizonte, Minas Gerais, Brazil

Fax: +55 3134993321

E-mail: malamut@cdtn.br

\begin{abstract}
In this paper, we report a formulation for the exposure buildup factor by solving the one-dimensional photon transport equation in a heterogeneous slab by the $\operatorname{LTS}_{N}$ method, assuming the Klein-Nishina scattering kernel as the scattering differential cross-section as well as the multigroup model in the wavelength variable. We present numerical simulations and comparisons with available results in the literature for a multilayered slab composed of water, iron and lead. We also report an analytical solution to the exposure buildup factor by solving the photon transport equation in a rectangle applying the $\mathrm{LTS}_{N}$ nodal method.
\end{abstract}


Keywords: exposure buildup factor; Klein-Nishina scattering kernel; multigroup model; $\mathrm{LTS}_{N}$ nodal method.

Reference to this paper should be made as follows: Amaral Rodriguez, B.D., de Vilhena, M.T., Borges, V. and Malamut, C. (2007) 'The exposure buildup factor formulation in a slab and rectangle geometry by the $\mathrm{LTS}_{N}$ method', Int. J. Low Radiation, Vol. 4, No. 2, pp.102-116.

Biographical notes: Bárbara Denicol do Amaral Rodriguez received her Master's degree in Applied and Computational Mathematics from the Federal University of Rio Grande do Sul. She is a doctorate student at the Department of Mechanics Engineering, Federal University of Rio Grande do Sul. Her current research interests include medical physics, radiation protection and nuclear engineering. She has published one research paper in an international journal and some papers at conference proceedings.

Marco Túllio de Vilhena received his doctorate degree in Mechanical Engineering from the Federal University of Rio Grande do Sul in 1988. He is a Full Professor at the Pure and Applied Mathematics Department, Federal University of Rio Grande do Sul. His research is in the fields of applied mathematics, radiation protection, radiative transfer and integral transform. $\mathrm{He}$ is an I-A Researcher of CNPq (Conselho Nacional de Desenvolvimento Cientifico e Tecnológico). He has published more than a hundred papers at international journals, about two hundred at national conference proceedings, one book and several chapters of books.

Volnei Borges received his doctorate degree in Mechanical Engineering in 1994. He is currently an Adjunct Professor at the Nuclear Engineering Department, Federal University of Rio Grande do Sul. His current research interests include radiation protection and medical physics. He has published research papers at national and international journals and conference proceedings.

Carlos Malamut received his doctorate degree in Physics from Pontifícia Universidade Católica do Rio de Janeiro, Brazil in 1987. He is currently Assistant to the Director of Research and Development of the Brazilian Nuclear Energy Commission located in Rio de Janeiro, Brazil. His current research interests include modelling in radiation transport, microdosimetry and tumour growth. He is the Vice President of Associação Brasileira de Física Médica, the Brazilian medical physics association.

\section{Introduction}

In the last decade, the $\mathrm{LTS}_{N}$ method, which solves, analytically, the discrete ordinates equation $\left(\mathrm{S}_{N}\right.$ equation) in a slab by the Laplace transform technique, has made an appearance in related literature. The main idea consists of the following steps: application of the Laplace transform technique to the set of $S_{N}$ equations, solution of the resulting algebraic equation by the matrix diagonalisation approach and inversion of the transformed angular flux by standard results of the Laplace transform theory. Here, analytical solution means that no approximation is made along the solution derivation. This methodology has been applied to a broad class of transport and radiative transfer 
problems. Some situations in which this methodology appears are the following: a general analytical approach to the one-group, one-dimensional transport equation by Barichello and Vilhena (1993); determination of the criticality parameters in heterogeneous slabs by the $\mathrm{LTS}_{N}$ method by Borges and Derivi (2001); the $\mathrm{LTS}_{N}$ method: a new analytical approach to solve the neutron transport equation by Vilhena and Barichello (1991); an analytical solution to the multigroup slab geometry discrete ordinates problems by Vilhena and Barichello (1995); extension of the $\mathrm{LTS}_{N}$ formulation for discrete ordinates problems without azimuthal symmetry by Segatto and Vilhena (1994); a new iterative method to solve the radiative transfer equation by Vilhena and Segatto (1996); the $\mathrm{LTS}_{N}$ solution for radiative transfer problems without azimuthal symmetry with severe anisotropy by Brancher et al. (1999); analytical solution of the discrete ordinates problem by the decomposition method by Vargas and Vilhena (1997); a closed-form solution for the one-dimensional radiative conductive problem by the decomposition and $\operatorname{LTS}_{N}$ methods by Vargas and Vilhena (1998); a closed-form solution to one-dimensional linear and non-linear radiative transfer problems by Vilhena and Barichello (1999); inverse problems for estimating bottom boundary conditions of natural waters in engineering by Velho et al. (2003); determining source term and boundary conditions in hydrological optics by Retamoso et al. (2001); estimation of boundary conditions in hydrologic optics by Retamoso et al. (2002); determination of the effective multiplication factor in a slab by the $\mathrm{LTS}_{N}$ method by Batistela et al. (1999); criticality by the $\operatorname{LTS}_{N}$ method by Batistela et al. (1997); recent advances in the $\mathrm{LTS}_{N}$ method for criticality calculations in slab geometry by Orengo et al. (2004); the $\operatorname{LTS}_{N}$ solution to the neutron transport equation in spherical geometry by Vasques et al. (2003); particle transport in the 1-D diffusive atomic mix limit by Larsen et al. (2005); and the convergence of the $\mathrm{LTS}_{N}$ method was proved by Pazos and Vilhena (1999; 2000). On the other hand, recently, the $\mathrm{LTS}_{N}$ method has been applied to the solution of the multidimensional $S_{N}$ nodal equations in cartesian geometry by Hauser (2002), Pazos et al. (2003) and Zabadal et al. (1995), and in the convex domain by Zabadal et al. (1997), considering one-group energy and isotropic scattering. To our knowledge, this methodology has not yet been applied to the solution of the transport equation assuming the Klein-Nishina scattering kernel and multigroup model for the wavelength variable.

Therefore, in the first part of this work, we step forward by solving the transport equation in a slab considering the Klein-Nishina scattering kernel and multigroup model by the $\mathrm{LTS}_{N}$ method. Bearing in mind the analyticity and the mentioned proved convergence of the $\mathrm{LTS}_{N}$ method, we are confident to emphasise that we can generate benchmark results in the exposure buildup factor by this methodology, controlling the accuracy by increasing $N$. In the second part, we report a two-dimensional $\mathrm{LTS}_{N}$ solution for a homogeneous rectangle assuming the Klein-Nishina scattering kernel and multigroup model. The main idea relies on the solution of the two one-dimensional $\mathrm{S}_{N}$ equations resulting from transverse integration of the $S_{N}$ equations in the rectangle by the $\mathrm{LTS}_{N}$ method, considering the leakage angular fluxes approximated exponentially, which allow us to determine a closed-form solution for the exposure buildup factor. Despite the lack of numerical validation, we are confident to affirm that the reported solution is actually a solution to the considered problem because the convergence of the $\operatorname{LTS}_{N}$ nodal solution discussed by Hauser et al. (2005) has been proven. Indeed, to reach our objectives, we organised the paper as follows: In Section 2, we present the $\mathrm{LTS}_{N}$ analytical solution to the exposure buildup factor in a slab assuming Klein-Nishina scattering kernel and multigroup model. In Section 3, we report numerical simulations 
and comparisons with available results in the literature. In Section 4, we display the two-dimensional $\mathrm{LTS}_{N}$ nodal solution for the exposure buildup factor considering the Klein-Nishina scattering kernel and multigroup model. Finally, in Section 5, we present a discussion about the methodology considered.

\section{The $\mathbf{L T S}_{N}$ solution}

In order to determine an analytical solution to the exposure buildup factor in a slab by the $\mathrm{LTS}_{N}$ method, let us consider the following $\mathrm{S}_{N}$ problem:

$$
\begin{gathered}
\mu_{n} \frac{\partial}{\partial x} I_{j n}(x)+\mu_{l j} I_{j n}(x)=\frac{\Delta}{3} \sum_{l=0}^{L} \frac{2 l+1}{2} \times \\
\times \sum_{r=1}^{G} c_{r} \alpha k_{r j} P_{l}\left(1+\lambda_{r}-\lambda_{j}\right) P_{l}\left(\mu_{n}\right) \sum_{i=1}^{N} P_{l}\left(\mu_{i}\right) I_{r i}(x) \omega_{i},
\end{gathered}
$$

subject to vacuum boundary conditions, for $j=1: G, n=1: N$,

where:

$$
\begin{aligned}
& G= \text { the number of energy groups (wavelengths) } \\
& N= \text { the Gaussian quadrature's order } \\
& \mu_{n}= \text { the roots of Legendre polynomial, ordered in decreasing } \\
& \text { fashion: }-1<\mu_{N}<\ldots<\mu_{\frac{N}{2}+1}<0<\mu_{\frac{N}{2}}<\ldots<\mu_{1}<1, \\
& \omega_{i}=\text { the weights of Gaussian quadrature } \\
& \mu_{l j}=\text { the linear attenuation coefficient, } \\
& I_{j n}(x)=I\left(x, \lambda_{j}, \mu_{n}\right)=\text { the angular flux at } \mu_{n} \text { direction for the } j \text {-th group } \\
& k_{r j}=k\left(\lambda_{r}, \lambda_{j}\right)=\text { Klein-Nishina scattering kernel, defined as follows: } \\
& k\left(\lambda_{r}, \lambda_{j}\right)=\frac{3}{8} \frac{\lambda_{r}}{\lambda_{j}}\left(\frac{\lambda_{r}}{\lambda_{j}}+\frac{\lambda_{j}}{\lambda_{r}}-\sin ^{2} \theta\right) .
\end{aligned}
$$

Following the idea of the $\mathrm{LTS}_{N}$ method, we begin applying the Laplace transform technique to Equation (1), with the resulting linear algebraic system:

$$
\begin{gathered}
s \overline{I_{j n}(s)}+\frac{\mu_{l j}}{\mu_{n}} \overline{I_{j n}(s)}-\frac{\Delta}{3 \mu_{n}} \sum_{l=0}^{L} \frac{2 l+1}{2} \sum_{r=1}^{G} c_{r} \alpha k_{r j} P_{l}\left(1+\lambda_{r}-\lambda_{j}\right) P_{l}\left(\mu_{n}\right) \\
\times \sum_{i=1}^{N} P_{l}\left(\mu_{i}\right) \omega_{i} \overline{I_{r i}(s)}=I_{j n}(0),
\end{gathered}
$$

for $j=1: G, n=1: N$, which can be recast in matrix form, as shown below:

$$
A_{j n}(s) \overline{I_{j n}(s)}=I_{j n}(0)+\overline{Z_{j-1}(s)} .
$$

Here, $\overline{I_{j n}(s)}$ is the $N$ component of the angular flux Laplace transformed vector and $I_{j n}(0)$ is the $N$ component of the angular flux vector at $x=0$. They have the form

$$
\overline{I_{j n}(s)}=\left[\overline{I_{j 1}(s)} \overline{I_{j 2}(s)} \ldots \overline{I_{j N}(s)}\right]^{T},
$$




$$
I_{j n}(0)=\left[I_{j 1}(0) I_{j 2}(0) \ldots I_{j N}(0)\right]^{T} .
$$

On the other hand, the entries of the $(N \times N)$ matrix $A_{j n}(s)$ are written as follows:

$$
a_{p q}= \begin{cases}s+\frac{\mu_{l j}}{\mu_{p}}-\frac{\Delta}{3 \mu_{p}} \sum_{l=0}^{L} \frac{2 l+1}{2} c_{j} \alpha k_{j j} P_{l}\left(\mu_{p}\right) P_{l}\left(\mu_{p}\right) \omega_{q} & \text { se } p=q \\ -\frac{\Delta}{3 \mu_{p}} \sum_{l=0}^{L} \frac{2 l+1}{2} c_{j} \alpha k_{j j} P_{l}\left(\mu_{p}\right) P_{l}\left(\mu_{q}\right) \omega_{q} & \text { se } p \neq q\end{cases}
$$

and the scattering term reads

$$
\overline{Z_{j-1}(s)}=\sum_{i=1}^{j-1} H_{i} \overline{I_{i n}(s)}
$$

where the components of constant matrix $H_{i}$ are given by the following:

$$
h_{p q}= \begin{cases}\frac{\Delta}{3 \mu_{p}} \sum_{l=0}^{L} \frac{2 l+1}{2} c_{i} \alpha k_{i j} P_{l}\left(1+\lambda_{i}-\lambda_{j}\right) P_{l}\left(\mu_{p}\right) P_{l}\left(\mu_{p}\right) \omega_{q} & \text { se } p=q \\ -\frac{\Delta}{3 \mu_{p}} \sum_{l=0}^{L} \frac{2 l+1}{2} c_{i} \alpha k_{i j} P_{l}\left(1+\lambda_{i}-\lambda_{j}\right) P_{l}\left(\mu_{p}\right) P_{l}\left(\mu_{q}\right) \omega_{q} & \text { se } p \neq q .\end{cases}
$$

Bearing in mind that for the Klein-Nishina scattering kernel the wavelength ranges from $\lambda_{0}$ to $\lambda_{0}+2$ ( $\lambda_{0}$ is the wavelength of the slab incoming radiation), we discretise, without loss of generality, this interval in five sub-intervals, meaning five groups, with the main feature that the first group (Group 1) corresponds to the sub-interval with the shortest wavelength and higher energy and Group 5, to the sub-interval with the longest wavelength and lowest energy.

Solving Equation (4) recursively for increasing wavelength ( $j$ from 1 to 5), due to the down-scattering, we come out with the result:

$$
\overline{I_{j n}(s)}=\left[A_{j}(s)\right]^{-1} I_{j n}(0)+\left[A_{j}(s)\right]^{-1} \overline{Z_{j-1}(s)} .
$$

Making the Laplace inversion of the above ansatz, we have

$$
I_{j n}(x)=\mathcal{L}^{-1}\left\{\left[A_{j n}(s)\right]^{-1} I_{j n}(0)\right\}+\mathcal{L}^{-1}\left\{\left[A_{j n}(s)\right]^{-1} \overline{Z_{j-1}(s)}\right\},
$$

which by the Heaviside expansion technique can be recast as follows:

$$
I_{j n}(x)=\sum_{k=1}^{j n} \beta_{k} e^{s_{k} x} I_{j n}(0)+Z_{j-1}(x) * \mathcal{L}^{-1}\left\{\left[A_{j n}(s)\right]^{-1}\right\},
$$

where:

$$
\beta_{k}=\left.\frac{\operatorname{Adj}\left(A_{j n}(s)\right)}{\frac{d}{d s}\left[\operatorname{det}_{j n}(s)\right]}\right|_{s=s_{k}}
$$


and the values of $s_{k}$ are the roots of the characteristics polynomial of the $A_{j n}(s)$ matrix. Here, a star denotes convolution. The exponential character of the generic method combined with the fact that the $s_{k}$ parameters increase in magnitude with $N$ implies that this formulation, in the proposed form, is not appropriate to solving large thickness transport problem. Fortunately, this difficulty was suppressed by introducing the ensuing modification for the basis space solution:

$$
I_{j n}(x)=\left(\sum_{k=I_{s<>0}}^{j n} \beta_{k} e^{-s_{k}(a-x)}+\sum_{k=I_{s k<0}}^{j n} \beta_{k} e^{s_{k} x}\right) I_{j n}^{*}(0)+Z_{j-1}(x) * \mathcal{L}^{-1}\left\{\left[A_{j}(s)\right]^{-1}\right\},
$$

where $I_{j n}^{*}(0)=C(a) \times I_{j n}(0)$ is the $N$ component modifying column vector and $C(a)$ reads as follows:

$$
C(a)=\left(\sum_{k=1_{s_{k}>0}}^{j n} \beta_{k} e^{-s_{k} a}+\sum_{k=s_{s_{k}<0}}^{j n} \beta_{k}\right)^{-1} .
$$

We determine the new arbitrary constant $I_{j n}^{*}(0)$ applying the boundary conditions.

The generalisation of the $\mathrm{LTS}_{N}$ solution for a heterogeneous slab assumes the Klein-Nishina scattering kernel and multigroup model is done in a straightforward manner. Indeed, we apply the $\mathrm{LTS}_{N}$ solution to each sublayer and evaluate the integration constants applying the boundary and interface conditions. This procedure leads to the following result for an arbitrary slab in the domain depicted in Figure 1:

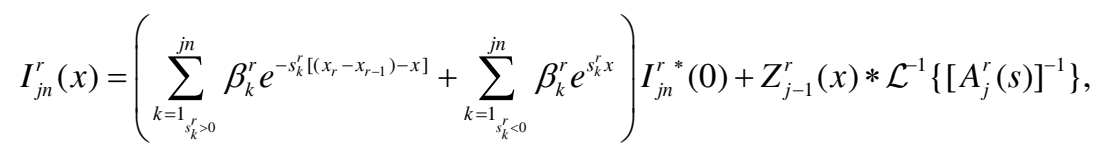

where $0<x<x_{R}-x_{R-1}, r=1: R$ and

$$
\begin{gathered}
I_{j n}^{r}(x)=\left[I_{11}^{r}(x) \ldots I_{1 N}^{r}(x) \ldots I_{j 1}^{r}(x) \ldots I_{j N}^{r}(x)\right]^{T}, \\
I_{j n}^{r}(0)=\left[I_{11}^{r}(0) \ldots I_{1 N}^{r}(0) \ldots I_{j 1}^{r}(0) \ldots I_{j N}^{r}(s)\right]^{T} .
\end{gathered}
$$

Here $I_{j n}^{r}(x)$ is the angular flux for the generic slab $r$.

Figure 1 Multilayered domain

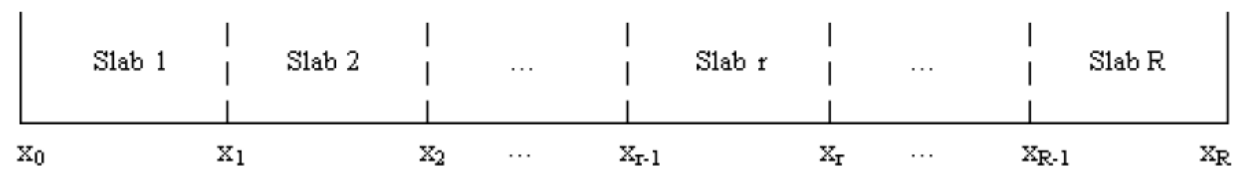

\section{Numerical results for the one-dimensional problem}

In order to illustrate the aptness of the discussed methodology to solve transport problems in a slab considering the Klein-Nishina scattering kernel and multigroup model, in the sequel we report numerical simulations for the discussed exposure buildup factor. To 
reach this goal, we evaluate the exposure buildup factor defined according to Fitzgerald et al. (1967) as the sum of the product of the attenuation coefficient of the air with the scalar flux for all radiations, including the incident flux, divided by the attenuation coefficient of the air for the incident flux multiplied by the incident scalar flux, shown as follows:

$$
B_{e}(x)=\frac{\sum_{i=0}^{j} \mu_{l}^{a i r}\left(\lambda_{i}\right) \varphi_{i}\left(x, \lambda_{i}\right)}{\mu_{l}^{a i r}\left(\lambda_{0}\right) \varphi_{0}\left(x, \lambda_{0}\right)} .
$$

Here, the subscript index 0 indicates the incident flux, $\mu_{l}^{\text {air }}\left(\lambda_{i}\right)$ is the attenuation coefficient of the air for the wavelength $\lambda_{i}, \mu_{l}^{\text {air }}\left(\lambda_{0}\right)$ is the attenuation coefficient of the air for the incident flux (for wavelength $\lambda_{0}$ ), $\varphi_{i}\left(x, \lambda_{i}\right)$ is the scalar flux for the wavelength $\lambda_{i}$ and $\varphi_{0}\left(x, \lambda_{0}\right)$ is the incident scalar flux. In what follows, we present numerical results for three problems.

Problem 1 Let us consider a multilayered slab with two regions, composed of water $\left(\mu_{l j}=0.0707 \mathrm{~cm}^{2} / \mathrm{g}, \mathrm{mfp}=1.0\right)$ and lead $\left(\mu_{l j}=0.06848 \mathrm{~cm}^{2} / \mathrm{g}, \mathrm{mfp}=4.0\right.$, $5.0,10.0,20.0,30.0$ and 40.0$)$ and under vacuum boundary conditions.

In Table 1 we present the $\mathrm{LTS}_{N}$ numerical simulations for the exposure buildup factor and comparisons with the ones (EGS ${ }_{4}$ results) attained by Hirayama and Shin (1998). Bearing in mind that $\mathrm{EGS}_{4}$ results are generated for the one-group model, given a closer look to the results in Table 1, we promptly realise a good coincidence. To underline the proved convergence of the $\operatorname{LTS}_{N}$ method in Table 2, we display the numerical convergence of the $\mathrm{LTS}_{N}$ results for increasing $N$. In fact, observing the results for $N=14$ and $N=16$, we notice a coincidence of six significant digits. Recalling the character of the solution, in the sense that no approximation is made along its derivation, except for the round-off error, this means that we may affirm that $B_{e}(x)=2.30$ and $B_{e}(x)=3.57$ are the exact results for Problem 1 and, consequently, are benchmark results for Problem 1. We bolster this affirmative recalling the proved convergence of the $\mathrm{LTS}_{N}$ method.

Table 1 Numerical exposure buildup factor simulations in water and lead composition

\begin{tabular}{lcc}
\hline & \multicolumn{2}{c}{ Water 1.0 mfp + Lead } \\
\cline { 2 - 3 }$M f p$ & LTS $_{16}$ & EGS $_{4}$ \\
\hline 4.0 & 2.30 & 2.31 \\
5.0 & 2.07 & 2.08 \\
10.0 & 3.57 & 3.59 \\
20.0 & 5.29 & 5.31 \\
30.0 & 6.77 & 6.79 \\
40.0 & 8.26 & 8.27 \\
\hline
\end{tabular}

Problem 2 To check the influence of the attenuation coefficient on the exposure buildup factor solution, let us consider the two-layered slab composed of water $\left(\mu_{l j}=0.0707 \mathrm{~cm}^{2} / \mathrm{g}, \mathrm{mfp}=1.0\right)$ and iron $\left(\mu_{l j}=0.0596 \mathrm{~cm}^{2} / \mathrm{g}\right.$, $\operatorname{mfp}=4.0,5.0,10.0,20.0,30.0$ and 40.0) and under vacuum boundary conditions. 
Table $2 \quad \mathrm{LTS}_{N}$ numerical convergence

\begin{tabular}{lcc}
\hline$N$ & $4 m f p$ & $10 m f p$ \\
\hline 2 & 2.29043291 & 3.56575823 \\
4 & 2.29124721 & 3.56773931 \\
6 & 2.29593785 & 3.56981458 \\
8 & 2.29921456 & 3.57019857 \\
12 & 2.30013541 & 3.57022344 \\
14 & 2.30014775 & 3.57022455 \\
16 & 2.30014786 & 3.57022457 \\
\hline
\end{tabular}

Table 3 Numerical exposure buildup factor simulations in water and iron composition

\begin{tabular}{lcc}
\hline & \multicolumn{2}{c}{ Water 1.0 mfp + Iron } \\
\cline { 2 - 3 }$m f p$ & $L T S_{16}$ & $E G S_{4}$ \\
\hline 4.0 & 4.99 & 5.01 \\
5.0 & 6.21 & 6.23 \\
10.0 & 13.9 & 13.9 \\
20.0 & 36.3 & 36.3 \\
30.0 & 67.6 & 67.5 \\
40.0 & 101 & 101 \\
\hline
\end{tabular}

Problem 3 Let us consider a heterogeneous slab with two regions, composed of lead $\left(\mu_{l j}=0.06848 \mathrm{~cm}^{2} / \mathrm{g}, \mathrm{mfp}=1.0\right)$ and iron $\left(\mu_{l j}=0.0596 \mathrm{~cm}^{2} / \mathrm{g}, \mathrm{mfp}=4.0\right.$, $5.0,10.0,20.0,30.0$ and 40.0) and under vacuum boundary conditions.

Table 4 Numerical exposure buildup factor simulations in lead and iron composition

\begin{tabular}{lcc}
\hline & \multicolumn{3}{c}{ Lead 1.0 mfp + Iron } \\
\cline { 2 - 3 }$m f p$ & LTS & $E G S_{4}$ \\
\hline 4.0 & 4.87 & 4.86 \\
5.0 & 6.34 & 6.28 \\
10.0 & 15.4 & 15.3 \\
20.0 & 41.5 & 41.4 \\
30.0 & 78.4 & 78.3 \\
40.0 & 118 & 117 \\
\hline
\end{tabular}

From the analysis of the results encountered for the above problems, we promptly realise a good agreement between the $\mathrm{LTS}_{16}$ and EGS 4 results. Bearing in mind the previously mentioned $\mathrm{LTS}_{16}$ results accuracy in Problem 1, we can also emphasise, supported by the same arguments, that the LTS $_{16}$ results encountered for the exposure buildup factor are also benchmark results. We must also mention that we have done all the calculations in an AMD Athlon $1700(1.4 \mathrm{GHz})$ microcomputer. Furthermore, the maximum computational time observed to generate all the results in each table is $90 \mathrm{~s}$. 


\section{The $\operatorname{LTS}_{N}$ nodal solution in a rectangle}

Let us consider the two-dimensional $\mathrm{S}_{N}$ nodal problem assuming the Klein-Nishina scattering kernel and multigroup model:

$$
\begin{gathered}
\mu_{n} \frac{\partial}{\partial x} I_{j n}(x, y)+\eta_{n} \frac{\partial}{\partial y} I_{j n}(x, y)+\mu_{l j} I_{j n}(x, y)= \\
=\frac{\Delta}{3} \sum_{l=0}^{L} \frac{2 l+1}{2} \sum_{r=1}^{G} c_{r} \alpha k_{r j} P_{l}\left(1+\lambda_{r}-\lambda_{j}\right) P_{l}\left(\mu_{n}\right) \sum_{i=1}^{N} P_{l}\left(\mu_{i}\right) I_{r i}(x, y) \omega_{i},
\end{gathered}
$$

subject to vacuum boundary conditions in a rectangle $0 \leq x \leq a$ and $0 \leq y \leq b$. Here $j=1: G, n=1: N, N=\frac{M(M+2)}{2}$ is the cardinality of the discrete ordinates set (number of discrete directions), $M$ represents the order of the angular quadrature, $G$ is the number of energy groups (wavelengths), $\mu_{l j}$ is the linear attenuation coefficient, $I_{j n}(x, y)=I\left(x, y, \lambda_{j}, \Omega_{n}\right)$ is the angular flux at the discrete direction $\Omega_{n}=\left(\mu_{n}, \eta_{n}\right)$ for the $j$-th group, the values of $\omega_{i}$ are the Lewis and Miller (1984) quadrature weights and $k_{r j}=k\left(\lambda_{r}, \lambda_{j}\right)$ is the Klein-Nishina scattering kernel defined by Equation (2).

To construct the $\operatorname{LTS}_{N}$ nodal solution for Problem (19), we begin performing the transverse integration of this equation. This procedure yields to the set of the ensuing two coupled $\mathrm{S}_{N}$ equations:

$$
\begin{gathered}
\eta_{n} \frac{d}{d y} I_{j n y}(y)+\frac{\mu_{n}}{a}\left[I_{j n}(a, y)-I_{j n}(0, y)\right]+\mu_{l j} I_{j n y}(y)= \\
=\frac{\Delta}{3} \sum_{l=0}^{L} \frac{2 l+1}{2} \sum_{r=1}^{G} c_{r} \alpha k_{r j} P_{l}\left(1+\lambda_{r}-\lambda_{j}\right) P_{l}\left(\mu_{n}\right) \sum_{i=1}^{N} P_{l}\left(\mu_{i}\right) I_{r i y}(y) \omega_{i},
\end{gathered}
$$

for $j=1: G, n=1: N$. Here $I_{j n}(a, y)$ and $I_{j n}(0, y)$ are the angular fluxes exiting at the boundary and the average angular flux is written as follows:

$$
\begin{aligned}
& I_{j n y}(y)=\frac{1}{a} \int_{0}^{a} I_{j n}(x, y) d x . \\
& \mu_{n} \frac{d}{d x} I_{j n x}(x)+\frac{\eta_{n}}{b}\left[I_{j n}(x, 0)-I_{j n}(x, b)\right]+\mu_{l j} I_{j n x}(x)= \\
& =\frac{\Delta}{3} \sum_{l=0}^{L} \frac{2 l+1}{2} \sum_{r=1}^{G} c_{r} \alpha k_{r j} P_{l}\left(1+\lambda_{r}-\lambda_{j}\right) P_{l}\left(\mu_{n}\right) \sum_{i=1}^{N} P_{l}\left(\mu_{i}\right) I_{r i x}(x) \omega_{i},
\end{aligned}
$$

for $j=1: G, n=1: N$. Here $I_{j n}(x, b)$ and $I_{j n}(x, 0)$ are the angular fluxes exiting at the boundary and the average angular flux is written as follows:

$$
I_{j n x}(x)=\frac{1}{b} \int_{0}^{b} I_{j n}(x, y) d y .
$$

At this point we are in a position to apply the $\mathrm{LTS}_{N}$ method. Indeed, we begin applying the Laplace transform technique in Equation (20). This procedure yields the following: 


$$
\begin{gathered}
s \overline{I_{j n y}(s)}+\frac{\mu_{l j}}{\eta_{n}} \overline{I_{j n y}(s)}-\frac{\Delta}{3 \eta_{n}} \sum_{l=0}^{L} \frac{2 l+1}{2} \sum_{r=1}^{G} c_{r} \alpha k_{r j} P_{l}\left(1+\lambda_{r}-\lambda_{j}\right) P_{l}\left(\mu_{n}\right) \times \\
\times \sum_{i=1}^{N} P_{l}\left(\mu_{i}\right) \overline{I_{r i y}(s)} \omega_{i}=I_{j n y}(0)-\frac{\mu_{n}}{a \eta_{n}}\left[\overline{I_{j n}(a, s)}-\overline{I_{j n}(0, s)}\right],
\end{gathered}
$$

for $j=1: G$ and $n=1: N$, which can be recast in matrix form as follows:

$$
\left(s I-B_{j n y}\right) \overline{I_{j n y}(s)}=I_{j n y}(0)+\overline{Z_{(j-1) y}(s)}+\overline{S_{j n y}(s)} .
$$

Here $\overline{I_{j n y}(s)}$ is the $N$ component of the angular flux Laplace transformed vector in the $y$ variable and $\overline{I_{j n y}(0)}$ is the $N$ component of the angular flux vector in the $y$ variable at $y=0$. They have the form

$$
\begin{aligned}
& \overline{I_{j n y}(s)}=\left[\overline{I_{j 1 y}(s)} \overline{I_{j 2 y}(s)} \ldots \overline{I_{j N y}(s)}\right]^{T}, \\
& \overline{I_{j n y}(0)}=\left[I_{j 1 y}(0) I_{j 2 y}(0) \ldots I_{j N y}(0)\right]^{T} .
\end{aligned}
$$

On the other hand, the components of matrix $B_{j n y}$ are given by

$$
b_{y}(p, q)= \begin{cases}-\frac{\mu_{l j}}{\eta_{p}}+\frac{\Delta}{3 \eta_{p}} \sum_{l=0}^{L} \frac{2 l+1}{2} c_{j} \alpha k_{j j} P_{l}\left(\mu_{p}\right) P_{l}\left(\mu_{p}\right) \omega_{q} & \text { se } p=q \\ \frac{\Delta}{3 \eta_{p}} \sum_{l=0}^{L} \frac{2 l+1}{2} c_{j} \alpha k_{j j} P_{l}\left(\mu_{p}\right) P_{l}\left(\mu_{q}\right) \omega_{q} & \text { se } p \neq q\end{cases}
$$

and the scattering term reads

$$
\overline{Z_{(j-1) y}(s)}=\sum_{i=1}^{j-1} H_{i y} \overline{I_{i n y}(s)},
$$

where the entries of constant matrix $H_{\text {iy }}$ are written as follows:

$$
h_{y}(p, q)= \begin{cases}\frac{\Delta}{3 \eta_{p}} \sum_{l=0}^{L} \frac{2 l+1}{2} c_{i} \alpha k_{i j} P_{l}\left(1+\lambda_{i}-\lambda_{j}\right) P_{l}\left(\mu_{p}\right) P_{l}\left(\mu_{p}\right) \omega_{q} & \text { se } p=q \\ -\frac{\Delta}{3 \eta_{p}} \sum_{l=0}^{L} \frac{2 l+1}{2} c_{i} \alpha k_{i j} P_{l}\left(1+\lambda_{i}-\lambda_{j}\right) P_{l}\left(\mu_{p}\right) P_{l}\left(\mu_{q}\right) \omega_{q} & \text { se } p \neq q .\end{cases}
$$

The vector $\overline{S_{j n y}(s)}$ has the generic component

$$
S_{j i y}(s)=\frac{\mu_{i}}{a \eta_{i}}\left[\overline{I_{j i}(a, s)}-\overline{I_{j i}(0, s)}\right] .
$$

A similar procedure in the $x$ variable leads to the ensuing linear algebraic system:

$$
\begin{gathered}
s \overline{I_{j n x}(s)}+\frac{\mu_{l j}}{\mu_{n}} \overline{I_{j n x}(s)}-\frac{\Delta}{3 \mu_{n}} \sum_{l=0}^{L} \frac{2 l+1}{2} \sum_{r=1}^{G} c_{r} \alpha k_{r j} P_{l}\left(1+\lambda_{r}-\lambda_{j}\right) P_{l}\left(\mu_{n}\right) \times \\
\times \sum_{i=1}^{N} P_{l}\left(\mu_{i}\right) \overline{I_{r i x}(s)} \omega_{i}=I_{j n x}(0)-\frac{\eta_{n}}{b \mu_{n}}\left[\overline{I_{j n}(s, b)}-\overline{I_{j n}(s, 0)}\right],
\end{gathered}
$$


which again can be recast in the matrix form as follows:

$$
\left(s I-A_{j n x}\right) \overline{I_{j n x}(s)}=I_{j n x}(0)+\overline{Z_{(j-1) x}(s)}+\overline{S_{j n x}(s)} .
$$

Here $\overline{I_{j n x}(s)}$ is the $N$ component of the angular flux Laplace transformed vector in the $x$ variable and $I_{j n x}(0)$ is the $N$ components of the angular flux vector in the $x$ variable at $x=0$. They have the form

$$
\begin{aligned}
\overline{I_{j n x}(s)} & =\left[\overline{I_{j 1 x}(s) I_{j 2 x}(s)} \ldots \overline{I_{j N x}(s)}\right]^{T}, \\
I_{j n x}(0) & =\left[I_{j 1 x}(0) I_{j 2 x}(0) \ldots I_{j N x}(0)\right]^{T} .
\end{aligned}
$$

On the other hand, the entries of matrix $A_{j n x}$ are written as follows:

$$
a_{x}(p, q)=\left\{\begin{array}{cc}
-\frac{\mu_{l j}}{\mu_{p}}+\frac{\Delta}{3 \eta_{p}} \sum_{l=0}^{L} \frac{2 l+1}{2} c_{i} \alpha k_{j j} P_{l}\left(\mu_{p}\right) P_{l}\left(\mu_{p}\right) \omega_{q} & \text { se } p=q \\
\frac{\Delta}{3 \mu_{p}} \sum_{l=0}^{L} \frac{2 l+1}{2} c_{j} \alpha k_{j j} P_{l}\left(\mu_{p}\right) P_{l}\left(\mu_{q}\right) \omega_{q} & \text { se } p \neq q .
\end{array}\right.
$$

and the scattering term reads

$$
\overline{Z_{(j-1) x}(s)}=\sum_{i=1}^{j-1} H_{i x} \overline{I_{i n x}(s)}
$$

where the constant matrix $H_{i x}$ have elements given by

$$
h_{x}(p, q)=\left\{\begin{array}{l}
\frac{\Delta}{3 \mu_{p}} \sum_{l=0}^{L} \frac{2 l+1}{2} c_{i} \alpha k_{i j} P_{l}\left(1+\lambda_{i}-\lambda_{j}\right) P_{l}\left(\mu_{p}\right) P_{l}\left(\mu_{p}\right) \omega_{q} \text { se } p=q \\
-\frac{\Delta}{3 \mu_{p}} \sum_{l=0}^{L} \frac{2 l+1}{2} c_{i} \alpha k_{i j} P_{l}\left(1+\lambda_{i}-\lambda_{j}\right) P_{l}\left(\mu_{p}\right) P_{l}\left(\mu_{q}\right) \omega_{q} \text { se } p \neq q,
\end{array}\right.
$$

and the vector $\overline{S_{j n x}(s)}$ reads

$$
\overline{S_{j i x}(s)}=\frac{\eta_{i}}{b \mu_{i}}\left[\overline{I_{j i}(s, b)}-\overline{I_{j i}(s, 0)}\right] .
$$

The $\operatorname{LTS}_{N}$ solution for Equations (25) and (33) are given by the following:

$$
\overline{I_{j n y}(s)}=\left(s I-B_{j n y}\right)^{-1}\left[I_{j n y}(0)+\overline{Z_{(j-1) y}(s)}+\overline{S_{j n y}(s)}\right]
$$

and

$$
\overline{I_{j n x}(s)}=\left(s I-A_{j n x}\right)^{-1}\left[I_{j n x}(0)+\overline{Z_{(j-1) x}(s)}+\overline{S_{j n x}(s)}\right] .
$$

Taking the Laplace inversion of the above ansatz we get,

$$
I_{j n y}(y)=\mathcal{L}^{-1}\left\{\left(s I-B_{j n y}\right)^{-1}\left[I_{j n y}(0)+\overline{Z_{(j-1) y}(s)}+\overline{S_{j n y}(s)}\right]\right\}
$$

and 


$$
I_{j n x}(x)=\mathcal{L}^{-1}\left\{\left(s I-A_{j n x}\right)^{-1}\left[I_{j n x}(0)+\overline{Z_{(j-1) x}(s)}+\overline{S_{j n x}(s)}\right]\right\} .
$$

Here, we do not proceed further to evaluate the Laplace inversion of Equations (42) and (43) because the inversion of these solutions has the same expression as the one in Equation (14). The explanation for this affirmative comes from the fact that the matrices $A_{j n x}$ and $B_{j n y}$ are also nondegenerate. To complete the solution, we have to determine the unknown leakage angular fluxes at boundary, namely, $I_{j n}(x, 0), I_{j n}(0, y), I_{j n}(x, b)$ and $I_{j n}(a, y)$. Following the work of Hauser (2002), which states that the exponential approximation gives the best results for the two-dimensional $\mathrm{LTS}_{N}$ nodal solution for deep penetration problems, we assume the ensuing approximation for the leakage angular fluxes:

$$
\begin{aligned}
& I_{j n}(x, 0)=F_{j n} e^{-\operatorname{sign}\left(\mu_{n}\right) \Lambda x} \\
& I_{j n}(0, y)=G_{j n} e^{-\operatorname{sign}\left(\eta_{n}\right) \Lambda y} \\
& I_{j n}(x, b)=O_{j n} e^{-\operatorname{sign}\left(\mu_{n}\right) \Lambda x} \\
& I_{j n}(a, y)=P_{j n} e^{-\operatorname{sign}\left(\eta_{n}\right) \Lambda y}
\end{aligned}
$$

where $\operatorname{sign}(\mu)$ denotes the signal function:

$$
\operatorname{sign}(\mu)=\left\{\begin{array}{r}
1 \text { if } \mu>0 \\
-1 \text { if } \mu>0
\end{array}\right.
$$

and $\Lambda$ represents the decay constant parameter, which has to be chosen a priori. In this work, we assume $\Lambda$, as Hauser (2002) did, as being the absorption cross-section given by:

$$
\Lambda=\sigma_{a}=\sigma_{t}-\sigma_{s}
$$

The functions $\operatorname{sign}\left(\mu_{n}\right)$ and $\operatorname{sign}\left(\eta_{n}\right)$, which appear in Equations (44)-(47), guarantee that the approximated angular fluxes will decay for any discrete direction. Replacing (44)-(47) in Equations (42) and (43), the $x$ - and $y$-averaged angular fluxes solutions are complete after the Laplace transform inversion. Applying the boundary conditions, we determine the integration constants and consequently the two-dimensional $\operatorname{LTS}_{N}$ nodal solution is well determined. Once the averaged angular fluxes are known, the closed-form solution for the two-dimensional exposure buildup factor is given by Equation (18), done by just replacing $\varphi(x, \lambda)$ with $\varphi(x, y, \lambda)$.

\section{Conclusion}

Concluding, we would like to point out our confidence that we hit our objective in this work because we succeeded in extending the $\mathrm{LTS}_{N}$ solution for problems in cartesian geometry, requiring the Klein-Nishina scattering kernel and multigroup model. This procedure allows us to derive a closed-form solution to the exposure buildup factor. To this point, we must recall that, before this work, the $\mathrm{LTS}_{N}$ solution was restricted to transport problems demanding isotropic and anisotropic scattering differential 
cross-section. Indeed, beginning our final analysis by looking at the first part of this work, we must emphasise that the $\mathrm{LTS}_{N}$ solution reported keeps the analytical feature, in the sense that no approximation is made along its derivation from the $S_{N}$ equations, except for the round-off error. Furthermore, the proved error-bound estimates and convergence by Pazos and Vilhena (1999; 2000), Hauser et al. (2005) assure that the LTS $_{N}$ solution converges to the exact solution when $N$ goes to infinity. We must also underline that the $\mathrm{LTS}_{N}$ method is quite general in the sense that it can now be applied to handle problems that demand the Klein-Nishina scattering kernel, which satisfies the error bound and convergence requirements. Therefore, we are emphasising that the mathematical analysis of the $\mathrm{LTS}_{N}$ is complete, regarding the issues of solution construction, error-bound estimates, convergence and results validation. Consequently, this method is a quite robust approach under either the mathematical or computational point of view to generate benchmark results. We reinforce this affirmative bearing in mind that, besides the analytical character of the solution, the $\mathrm{LTS}_{N}$ method solves transport problems that requires large $N(N \leq 2000)$ with a small computational time. On the other hand, concerning the second part, we begin by saying that the major claim refers to the issue of mathematical analysis that consists of the solution derivation presented in Section 2, as well as the proved error-bound estimates and convergence by Pazos and Vilhena $(1999 ; 2000)$ and Hauser et al. (2005). The justificative for this affirmative comes from the fact that the solution to the two-dimensional $\mathrm{S}_{N}$ nodal problem is reduced to the solution of a set of two one-dimensional $\mathrm{S}_{N}$ equations, which are quite similar to the ones validated in Section 1. Therefore, we believe that the mathematical analysis, somehow, compensates for the lack of results validation for the two-dimensional $\mathrm{LTS}_{N}$ nodal solution. Now, regarding the topic of analyticity, we must emphasise that the unique approximation made along the derivation of the $\mathrm{LTS}_{N}$ nodal solution was in the leakage angular flux at boundary. In addition, we find it relevant to comment that our analysis is restricted to the homogeneous rectangle, because we attain, in a straightforward manner, the solution for the heterogeneous rectangle proceeding likewise in the heterogeneous slab. Finally, pursuing our objective of searching for analytical solutions, we focus our future attention on the issue of extending the LTS $_{N}$ nodal solution for the three-dimensional problem in a heterogeneous parallelepiped assuming the discussed kernels. We also intend to complete the mathematical analysis concerning the issues of error-bound estimates and convergence. We hope to show by this procedure the aptness and robustness of the $\mathrm{LTS}_{N}$ method to solve a broad class of transport problems.

\section{Acknowledgements}

The first and second authors are gratefully indebted to CNPq (Conselho Nacional de Desenvolvimento Cientifíco e Tecnológico) for the partial financial support to this work. 


\section{References}

Barichello, L.B. and Vilhena, M.T. (1993) 'A general analytical approach to one group one dimensional transport equation', Kerntechnik, Vol. 58, No. 5, p.182.

Batistela, C.H., Vilhena, M.T. and Borges, V. (1997) 'Criticality by the $\mathrm{LTS}_{N}$ method', Journal of Nuclear Science and Technology, Vol. 34, p.603.

Batistela, C.H., Vilhena, M.T. and Borges, V. (1999) 'Determination of the effective multiplication factor in a slab by the $\mathrm{LTS}_{N}$ method', Annals of Nuclear Energy, Vol. 26, p.761.

Borges, V. and Derivi, A.G. (2001) 'Determination of the criticality parameters in heterogeneous slab by the $\mathrm{LTS}_{N}$ method', Proceedings of the International Conference on Computational Heat and Mass Transfer, Rio de Janeiro, Brazil.

Brancher, J., Segatto, C. and Vilhena, M.T. (1999) 'The $\mathrm{LTS}_{N}$ solution for radiative transfer problem without azimuthal symmetry with severe anisotropy', Journal of Quantitative Spectroscopy and Radiative Transfer, Vol. 62, p.743.

Fitzgerald, J.J., Brownell, G.L. and Mahoney, F.J. (1967) Mathematical Theory of Radiation Dosimetry, New York: Gordon and Breach.

Hauser, E.B. (2002) 'Solution of the two-dimensional neutron transport equation by the $\mathrm{LTS}_{N}$ method for high order of quadrature: LTS $_{N}$ 2D-Diag e LTS $_{N}$ 2D-DiagExp', DSc thesis, Mechanical Engineering Program, Federal University of Rio Grande do Sul, Porto Alegre, Brazil [in Portuguese].

Hauser, E.B., Pazos, R.P. and Vilhena, M.T. (2005) 'An error bound estimate and convergence of the nodal-LTS ${ }_{N}$ solution in a rectangle', Annals of Nuclear Energy, Vol. 32, p.1146.

Hirayama, H. and Shin, K. (1998) 'Application of the EGS4 Monte Carlo code to a study of multilayer Gamma-ray exposure buildup factors', Journal of Nuclear Science and Technology, Vol. 35, p.816.

Larsen, E.W., Vasques, R. and Vilhena, M.T. (2005) 'Particle transport in the 1-D diffusive atomic mix limit', Mathematics and Computation, Supercomputing, Reactor Physics and Nuclear and Biological Applications, France.

Lewis, E.E. and Miller, W.F. (1984) Computational Methods of Neutron Transport, New York: Wiley.

Orengo, G., Vilhena, M.T., Graça, C.O., Caldeira, A.D. and Gonçalves, G.A. (2004) 'Recent advances in the $\mathrm{LTS}_{N}$ method for criticality calculations in slab geometry', Annals of Nuclear Energy, Vol. 31, p.2195.

Pazos, R.P., Hauser, E.B. and Vilhena, M.T. (2003) 'Advances in the solution of three-dimensional nodal neutron transport equation', 11th International Conference on Nuclear Engineering, Tokyo.

Pazos, R.P. and Vilhena, M.T. (1999) 'Convergence of the LTS $_{N}$ method: approach of CO semi groups', Progress in Nuclear Energy, Vol. 34, No. 1, p.77.

Pazos, R.P. and Vilhena, M.T. (2000) 'Convergence in transport theory', Applied Numerical Mathematics, Vol. 30, p.79.

Retamoso, M., Velho, H. and Vilhena, M.T. (2001) 'Determining source term and boundary conditions in hydrological optics', 2nd International Conference on Computational Heat and Mass Transfer, Rio de Janeiro, Brazil.

Retamoso, M., Vilhena, M.T. and Velho, H. (2002) 'Estimation of boundary condition in hydrologic optics', Applied Numerical Mathematics, Vol. 40, p.87.

Segatto, C.F. and Vilhena, M.T. (1994) 'Extension of the $\mathrm{LTS}_{N}$ formulation for discrete ordinates problems without azimuthal symmetry', Annals of Nuclear Energy, Vol. 21, p.701.

Vargas, R. and Vilhena, M.T. (1997) 'Analytical solution of the discrete ordinates problem by the decomposition method', Annals of Nuclear Energy, Vol. 24, p.785. 
Vargas, R. and Vilhena, M.T. (1998) 'A closed-form solution for one-dimensional radiative conductive problem by the decomposition and $\mathrm{LTS}_{N}$ methods', Journal of Quantitative Spectroscopy and Radiative Transfer, Vol. 61, p.303.

Vasques, R., Segatto, C.F. and Vilhena, M.T. (2003) 'The $\mathrm{LTS}_{N}$ solution for the neutron transport equation in spherical geometry', Proceedings of the 18th International Conference on Transport Theory, Rio de Janeiro, p.265.

Velho, H., Retamoso, M. and Vilhena, M.T. (2003) 'Inverse problems for estimating bottom boundary conditions of natural waters in engineering', International Journal for Numerical Methods in Engineering, Vol. 54, p.1357.

Vilhena, M.T. and Barichello, L.B. (1991) 'The LTS $_{N}$ method: a new analytical approach to solve the neutron transport equation', Kerntechnik, Vol. 56, No. 5, p.334.

Vilhena, M.T. and Barichello, L.B. (1995) 'An analytical solution for the multi-group slab geometry discrete ordinates problems', Transport Theory and Statistical Physics, Vol. 24, p.1337.

Vilhena, M.T. and Barichello, L.B. (1999) 'A closed-form solution to one-dimensional linear and nonlinear radiative transfer problem', Hybrid Methods in Engineering, Vol. 1, p.1.

Vilhena, M.T. and Segatto, C.F. (1996) 'A new iterative method to solve the radiative transfer equation', Journal of Quantitative Spectroscopy and Radiative Transfer, Vol. 55, p.493.

Zabadal, J.S., Vilhena, M.T. and Barichello, L.B. (1995) 'Solution of the three-dimensional one-group discrete ordinates problem by the $\mathrm{LTS}_{N}$ method', Annals of Nuclear Energy, Vol. 22, p.131.

Zabadal, J.S., Vilhena, M.T. and Barichello, L.B. (1997) 'An analytical solution for the two-dimensional for discrete ordinate problem in a convex domain', Progress in Nuclear Energy, Vol. 31, No. 3, p.225. 